\title{
Reynolds-number dependence of turbulence enhancement on collision growth
}

\author{
Ryo Onishi ${ }^{1}$ and Axel Seifert ${ }^{2}$ \\ ${ }^{1}$ Center for Earth Information Science and Technology, Japan Agency for Marine-Earth Science and Technology, \\ 3173-25 Showa-machi, Kanazawa-ku, Yokohama Kanagawa 236-0001 Japan \\ ${ }^{2}$ Deutscher Wetterdienst, Offenbach, Germany \\ Correspondence to: Ryo Onishi (onishi.ryo@jamstec.go.jp)
}

Received: 9 January 2016 - Published in Atmos. Chem. Phys. Discuss.: 5 February 2016

Revised: 1 August 2016 - Accepted: 15 September 2016 - Published: 5 October 2016

\begin{abstract}
This study investigates the Reynolds-number dependence of turbulence enhancement on the collision growth of cloud droplets. The Onishi turbulent coagulation kernel proposed in Onishi et al. (2015) is updated by using the direct numerical simulation (DNS) results for the Taylormicroscale-based Reynolds number $\left(R e_{\lambda}\right)$ up to 1140 . The DNS results for particles with a small Stokes number $(S t)$ show a consistent Reynolds-number dependence of the socalled clustering effect with the locality theory proposed by Onishi et al. (2015). It is confirmed that the present Onishi kernel is more robust for a wider $S t$ range and has better agreement with the Reynolds-number dependence shown by the DNS results. The present Onishi kernel is then compared with the Ayala-Wang kernel (Ayala et al., 2008a; Wang et al., 2008). At low and moderate Reynolds numbers, both kernels show similar values except for $r_{2} \sim r_{1}$, for which the AyalaWang kernel shows much larger values due to its large turbulence enhancement on collision efficiency. A large difference is observed for the Reynolds-number dependences between the two kernels. The Ayala-Wang kernel increases for the autoconversion region $\left(r_{1}, r_{2}<40 \mu \mathrm{m}\right)$ and for the accretion region $\left(r_{1}<40\right.$ and $r_{2}>40 \mu \mathrm{m} ; r_{1}>40$ and $\left.r_{2}<40 \mu \mathrm{m}\right)$ as $R e_{\lambda}$ increases. In contrast, the Onishi kernel decreases for the autoconversion region and increases for the rain-rain self-collection region $\left(r_{1}, r_{2}>40 \mu \mathrm{m}\right)$. Stochastic collisioncoalescence equation (SCE) simulations are also conducted to investigate the turbulence enhancement on particle size evolutions. The SCE with the Ayala-Wang kernel (SCEAyala) and that with the present Onishi kernel (SCE-Onishi) are compared with results from the Lagrangian Cloud Simulator (LCS; Onishi et al., 2015), which tracks individual par-
\end{abstract}

ticle motions and size evolutions in homogeneous isotropic turbulence. The SCE-Ayala and SCE-Onishi kernels show consistent results with the LCS results for small $R e_{\lambda}$. The two SCE simulations, however, show different Reynolds-number dependences, indicating possible large differences in atmospheric turbulent clouds with large $R e_{\lambda}$.

\section{Introduction}

Several mechanisms have been proposed to explain the rapid growth of cloud droplets, which often result in fast rain initiation in the early stages of cloud development. Examples of these mechanisms include the turbulence-enhanced collision rate of cloud droplets (Falkovich and Pumir, 2007; Grabowski and Wang, 2013), turbulent entrainment (Blyth, 1993; Krueger et al., 1997), giant cloud condensation nuclei (Yin et al., 2000; Van Den Heever and Cotton, 2007), and turbulent dispersions of cloud droplets (Sidin et al., 2009). The first mechanism, which has received the most attention, has led to extensive research on particle collisions in turbulence (e.g., Sundaram and Collins, 1997; Wang et al., 2000; Saw et al., 2008; Onishi et al., 2009; Dallas and Vassilicos, 2011).

One direction taken by the research in this area is the simulation of collisional growth by solving the stochastic collision-coalescence equation (SCE). Such research relies on accurate collision-coalescence models, which consist of models for the collision kernel $K_{\mathrm{c}}\left(r_{1}, r_{2}\right)$ (where $r_{i}$ is the particle radius), the collision efficiency $E_{\mathrm{c}}\left(r_{1}, r_{2}\right)$, and the coalescence efficiency $E_{\text {coal }}\left(r_{1}, r_{2}\right)$. To consider the influence of turbulence, several turbulent collision models have 
been proposed. Saffman and Turner (1956) analytically derived a collision kernel model for particles with no inertia or with a very small Stokes number $\left(S t=\tau_{\mathrm{p}} / \tau_{\eta}\right.$, where $\tau_{\mathrm{p}}$ is the particle relaxation time and $\tau_{\eta}$ is the Kolmogorov time), while Abrahamson (1975) derived a model for $S t \gg 1$. For moderate Stokes numbers, i.e., $S t \sim 1$, one difficulty is the preferential motion of inertial particles. Inertial particles preferentially cluster in regions of low vorticity and high strain if $S t \ll 1$ (Maxey, 1987), and they cluster in a way that mimics the clustering of zero-acceleration points by the sweep-stick mechanism if $1 \lesssim S t \lesssim \tau_{\mathrm{p}} / T_{\mathrm{I}}$, where $T_{\mathrm{I}}$ is the integral timescale of the turbulence (Coleman and Vassilicos, 2009). This matters because clustering increases the mean collision rate (Sundaram and Collins, 1997). To quantify the clustering due to the preferential concentration effect, a model is formulated for finite-inertial particles. However, the model requires several empirical parameters that should be determined from reference data, e.g., results from a direct numerical simulation (DNS).

One serious problem is that the Reynolds-number dependence of turbulent collisions has not yet been clarified. In fact, many authors ignore the Reynolds-number dependence and assume a constant collision kernel regardless of the Reynolds number (e.g., Saffman and Turner, 1956; Derevyanko et al., 2008; Zaichik and Alipchenkov, 2009) or assume a convergence to a constant collision kernel with increasing Reynolds number (e.g., Ayala et al., 2008a). Onishi et al. (2013) observed that the clustering effect, and consequently the collision kernel, decreases as the Taylor-microscale-based Reynolds number $\left(R e_{\lambda}\right)$ increases for $S t=0.4$. Onishi and Vassilicos (2014) later clarified that the Reynolds-number dependence of the clustering effect for $1 / 3 \lesssim S t \lesssim 1$ is due to internal intermittency of the turbulence. Because a robust theoretical model for turbulent collision kernels is not yet available, we need empirical models for the investigation of turbulence enhancement on cloud development. As an example, the Ayala-Wang kernel (Ayala et al., 2008a; Wang et al., 2008) is a widely used turbulent kernel model.

Recently, Onishi et al. (2015) proposed an empirical kernel model based on DNS data for the wide range of $49 \leq R e_{\lambda} \leq$ 530, where $R e_{\lambda}$ is the Taylor-microscale-based Reynolds number. Onishi et al. (2015) also conducted stochastic and direct collision simulations to investigate the turbulence enhancement on drop size evolution. They investigated the energy dissipation $(\epsilon)$ dependence for the range of $100 \leq$ $\epsilon \leq 1000 \mathrm{~cm}^{2} \mathrm{~s}^{-3}$ and the $R e_{\lambda}$ dependence for the range of $66 \leq R e_{\lambda} \leq 206$. The results showed good agreement of the $\epsilon$ dependence between the stochastic simulations with the Ayala-Wang and Onishi kernels, but a significant discrepancy for the $R e_{\lambda}$ dependence between the two kernels. The discrepancy in $R e_{\lambda}$ dependence may become a critical issue for cloud simulations because $R e_{\lambda}$ is typically as large as $\mathrm{O}\left(10^{3-4}\right)$ in atmospheric turbulent clouds. However, Onishi et al. (2015) did not provide a detailed discussion on the difference of the Ayala-Wang and Onishi kernels in $R e_{\lambda}$ dependence.

This study, therefore, aims to compare the Ayala-Wang and Onishi kernels by focusing on their $R e_{\lambda}$ dependence. First, the Onishi kernel is updated by using the reference collision statistics obtained by the DNS for $R e_{\lambda}$ up to 1140 . The Ayala-Wang and the present Onishi kernel values are compared in detail. The SCE simulations with the Ayala-Wang and Onishi kernels are also compared with each other and with the reference results from the Lagrangian Cloud Simulator (LCS; Onishi et al., 2015), which tracks individual particle motions and size evolutions in homogeneous isotropic turbulence. The collision growth simulation with the LCS is conducted for $R e_{\lambda}$ up to 333 .

\section{Turbulent coagulation kernel models}

\subsection{Turbulent coagulation kernel}

The geometric collision frequency per unit volume between particles with radius $r_{1}$ and those with radius $r_{2}, N_{\mathrm{c}}\left(r_{1}, r_{2}\right)$, is expressed by the geometric collision kernel $K_{\mathrm{c}}\left(r_{1}, r_{2}\right)$ as

$N_{\mathrm{c}}\left(r_{1}, r_{2}\right)=K_{\mathrm{c}}\left(r_{1}, r_{2}\right) n_{\mathrm{p}, 1} n_{\mathrm{p}, 2}$,

where $n_{\mathrm{p}, i}$ is the number density of particles with radius $r_{i}$. The coagulation kernel $K_{\text {coag }}$ can be expressed by the combination of the geometric collision kernel, collision efficiency $E_{\mathrm{c}}$, and coalescence efficiency $E_{\mathrm{coal}}$ as

$K_{\text {coag }}\left(r_{1}, r_{2}\right)=E_{\text {coal }}\left(r_{1}, r_{2}\right) E_{\mathrm{c}}\left(r_{1}, r_{2}\right) K_{\mathrm{c}}\left(r_{1}, r_{2}\right)$.

The gravitational collision kernel describes the collisions due to the settling velocity difference in the form of

$K_{\mathrm{c}, \text { grav }}\left(r_{1}, r_{2}\right)=\pi R_{12}^{2}\left|V_{\infty 1}-V_{\infty 2}\right|$,

where $R_{12}\left(=r_{1}+r_{2}\right)$ is the collision radius and $V_{\infty i}$ is the gravitational particle settling velocity. Turbulence enlarges the geometric collision kernel, i.e., the turbulent geometric kernel $K_{\mathrm{c} \text {,turb }}$ is larger than $K_{\mathrm{c} \text {,grav }}$. Turbulence also enhances the coagulation kernel through enlarging $E_{\mathrm{c}}$. The turbulence enhancement on the collision efficiency, $\eta_{E}$, is defined as

$\eta_{E}\left(r_{1}, r_{2}\right)=\frac{E_{\mathrm{c}}\left(r_{1}, r_{2}\right)[\mathrm{T}]}{E_{\mathrm{c}}\left(r_{1}, r_{2}\right)[\mathrm{NoT}]}$,

where $[\mathrm{T}]$ and $[\mathrm{NoT}]$ indicate the turbulent flow case and the stagnant (non-turbulent) flow case, respectively.

It had been difficult to confidently discuss the collision efficiency in a turbulent flow until Ayala et al. (2007) developed a reliable superposition method, which iteratively solves the Stokes disturbance flows for a many-particle system. That superposition method is, however, computationally expensive due to its iteration procedure. Onishi et al. (2013) later developed a less costly method, named the binary-based superposition method (BiSM), which has been adopted in the 
LCS (Onishi et al., 2015). BiSM assumes that interactions via three or more particles are negligible. This dramatically reduces the computational cost but maintains reliability as long as the particle number concentration is small, as observed in atmospheric clouds.

Sundaram and Collins (1997) showed, by means of a DNS, that the preferential concentration of inertial particles, the so-called clustering effect, increases the collision frequency. The clustering effect is expressed in the spherical formulation derived by Wang et al. (1998) as

$$
K_{\mathrm{c}}\left(r_{1}, r_{2}\right)=2 \pi R_{12}^{2}\left\langle\left|w_{\mathrm{r}}\left(x=R_{12}\right)\right|\right\rangle g_{12}\left(x=R_{12}\right),
$$

where $\langle\ldots\rangle$ denotes an ensemble average, $\left|w_{\mathrm{r}}\left(x=R_{12}\right)\right|$ $\left(\left|w_{\mathrm{r}}\right|\right.$ hereafter) is the radial relative velocity at contact separation, and $g_{12}\left(x=R_{12}\right)$ ( $g_{12}$ hereafter) is the radial distribution function at contact separation and represents the clustering effect.

Droplet deformation and coalescence efficiency, which this study ignores, affect the collision growth of droplets with $r>100 \mu \mathrm{m}$, although such effects only become significant for droplets with $r>500 \mu \mathrm{m}$. It would, therefore, lead to some errors if extending the present results to such large droplets.

\subsection{Ayala-Wang model}

Ayala et al. (2008a) provided a parameterization for the turbulent geometric collision kernel of finite-inertia sedimenting droplets by proposing an empirical model for $g_{12}$ in addition to a theoretical model for $\left\langle\left|w_{\mathrm{r}}\right|\right\rangle$.

By following the expression by Chun et al. (2005), the clustering effect for a monodisperse suspension of sedimenting droplets is expressed as

$g_{11}=\left(\frac{\eta}{r}\right)^{C_{1}}$,

where $\eta$ is the Kolmogorov length. $C_{1}$ is a function of $S t$, $R e_{\lambda}$, and the non-dimensional parameter for gravity $V_{\infty} / v_{\eta}$ with the Kolmogorov velocity $v_{\eta}$. This parameterization was extended for a bidisperse system in a manner similar to that in Chun et al. (2005):

$g_{12}=\left(\frac{\eta^{2}+r_{\mathrm{d}}^{2}}{r_{\mathrm{L}}^{2}+r_{\mathrm{d}}^{2}}\right)^{C_{1} / 2}$,

where $r_{\mathrm{L}}=\max \left(r_{1}, r_{2}\right)$ and $C_{1}$ follows the same expression for the monodisperse case at $S t_{\max }=\max \left(S t_{1}, S t_{2}\right)=S t\left(r_{\mathrm{L}}\right)$, and $r_{\mathrm{d}}$ is a length scale of the acceleration diffusion experienced by the particles. When two particles in a pair are two different sizes, any fluid acceleration or gravity will induce a relative velocity. This effect yields a diffusion-like process in the system and tends to smooth out inhomogeneities in the particle pair concentration. Thus, $r_{\mathrm{d}}$ is larger for larger $\left|S t_{1}-S t_{2}\right|$ for the bidisperse case and a monodisperse suspension form is recovered for the case $r_{\mathrm{d}} \ll r_{\mathrm{L}}$. It should be noted for the discussion in Sect 4.4 that the $g_{12}$ model was designed to show maximum clustering at $S t \sim 1$ and a higher droplet clustering for larger $R e_{\lambda}$ (Ayala et al., 2008b).

In addition to the empirical $g_{12}$ model, Ayala et al. (2008a) developed a theory for $\left\langle\left|w_{\mathrm{r}}\right|\right\rangle$ that is applicable to inertial droplets sedimenting under gravity in a turbulent flow. The basic assumption was that the droplet relative trajectory is mostly determined by gravitational sedimentation. Following Dodin and Elperin (2002), they decomposed the radial relative velocity (between two particles falling under gravity in a homogeneous isotropic turbulent flow) into a random part $\xi$ caused by turbulent fluctuations and a deterministic part $h$ due to gravity:

$w_{\mathrm{r}}(\phi)=\xi(\phi)+h(\phi)$,

where the angle of contact, $\phi$, is measured from the gravity axis. The random variable $\xi(\phi)$ is assumed to be normally distributed with a standard deviation $\sigma(\phi)$.

Using $\sigma\left(\phi=90^{\circ}\right)$ to approximate $\sigma(\phi)$, they obtained

$\left\langle\left|w_{\mathrm{r}}\right|\right\rangle=\sqrt{\frac{2}{\pi}}\left(\sigma^{2}+\frac{\pi}{8}\left(\tau_{\mathrm{p}, 1}-\tau_{\mathrm{p}, 2}\right)^{2} g^{2}\right)^{1 / 2}$,

where $\sigma$ is expressed in terms of $\tau_{\mathrm{p}, i}, V_{\infty i}$ and flow parameters $u_{\mathrm{rms}}$ (the rms of the velocity fluctuations) in terms of $\epsilon$ and $R e_{\lambda}$.

\subsection{Onishi model}

\subsubsection{Model for $g_{12}$}

Onishi et al. (2015) proposed an original model for the clustering effect in monodisperse systems of non-sedimenting particles with Stokes' linear drag.

$g_{11}-1=\left\{\begin{array}{ll}A_{1} S t^{2} & \left(\equiv y_{1}\right)\left(\text { for } S t<S t_{a}\right) \\ A_{2} R e_{\lambda} S t^{-2} & \left(\equiv y_{2}\right)\left(\text { for } S t_{a} \leq S t\right)\end{array}\right.$,

where $A_{1}$ and $A_{2}$ were empirically determined to be 110 and 0.38 , respectively. The regime boundary $S t_{a}$ is $\left(A_{2} / A_{1}\right)^{1 / 4} R e_{\lambda}^{1 / 4}$. A tanh smoothing function, $z_{a}$, was employed to connect the two formulations in the equation as

$g_{11}-1=H\left(S t-S t_{a}\right) y_{1} z_{a}^{\alpha}+H\left(S t_{a}-S t\right) y_{2}\left(1-z_{a}\right)^{\alpha}$.

(Note that the Heaviside function was missing in Onishi et al., 2015.) Here,

$z_{a}(S t)=\frac{1}{2}\left(1-\tanh \frac{\log _{10} S t-\log _{10} S t_{a}}{C_{a}}\right)$,

where $C_{a}$ is parameterized as 
$C_{a}=a_{c} \operatorname{Re}_{\lambda}^{b_{c}}$.

In Eq. (11), $\alpha$ is parameterized as

$\alpha=\log _{2} a_{\alpha} R e_{\lambda}^{b_{\alpha}}$.

Onishi et al. (2015) determined the optimal values for the abovementioned empirical coefficients (i.e., $A_{1}, A_{2}, a_{c}, b_{c}$, $a_{\alpha}$, and $b_{\alpha}$ ) based on the dataset in Onishi et al. (2013); Onishi and Vassilicos (2014) for $S t \leq 1$.

If we limited the discussion for the autoconversion regime, i.e., $r<40 \mu \mathrm{m}$, the range $S t \leq 1$ would be enough for the typical energy dissipation rate $\epsilon \leq 1000 \mathrm{~cm}^{2} \mathrm{~s}^{-3}$ observed in atmospheric turbulent clouds. However, as clearly shown in Fig. 1, St can be as large as 10 for $r=100 \mu \mathrm{m}$ and $\epsilon=$ $1000 \mathrm{~cm}^{2} \mathrm{~s}^{-3}$. That is, in the discussion on the accretion process that describes the conversion from cloud to rain due to rain drops collecting cloud droplets, we need to deal with $S t>1$ as well.

Hence, this study modifies the parameterization in the original Onishi kernel to obtain better overall matching for a wider range of $S t$. After trial and error, we finally obtained a modification of the form of $C_{a}$ as

$C_{a}=\min \left(a_{c} R e_{\lambda}^{b_{c}}, a_{c 2} R e_{\lambda}^{b_{c 2}}\right)$.

We confirmed that this form with $a_{c}=0.046, b_{c}=0.36$, $a_{c 2}=0.094$, and $b_{c 2}=0.25$ leads to an improvement, as shown later in Sect. 4.2. The updated coefficients are summarized in Table 1.

To determine the clustering effect for bidisperse systems, the empirical formulation proposed by Zhou et al. (2001) is employed:

$g_{12}=1+\rho_{12}\left(g_{11}-1\right)^{1 / 2}\left(g_{22}-1\right)^{1 / 2}$,

where $\rho_{12}=2.6 \exp \left(-S t_{\max }\right)+$ $0.205 \exp \left(-0.0206 S t_{\max }\right) \frac{1}{2}\left[1+\tanh \left(S t_{\max }-3\right)\right]$.

The gravitational settling affects the clustering effect for large St particles. The parameterization here does not consider the gravity effect. This would lead to some error in collision statistics. However, the error was not significant in this study and the present parameterization worked well for predicting the turbulence enhancement in size evolutions due to collisional growth as in Sect. 4.5.

\subsubsection{Model for $\left\langle\left|w_{\mathbf{r}}\right|\right\rangle$}

Onishi et al. (2015) employed the model of Wang et al. (2000) for $\left\langle\left|w_{\mathrm{r}}\right|\right\rangle$, which was based on the model by Kruis and Kusters (1997), as

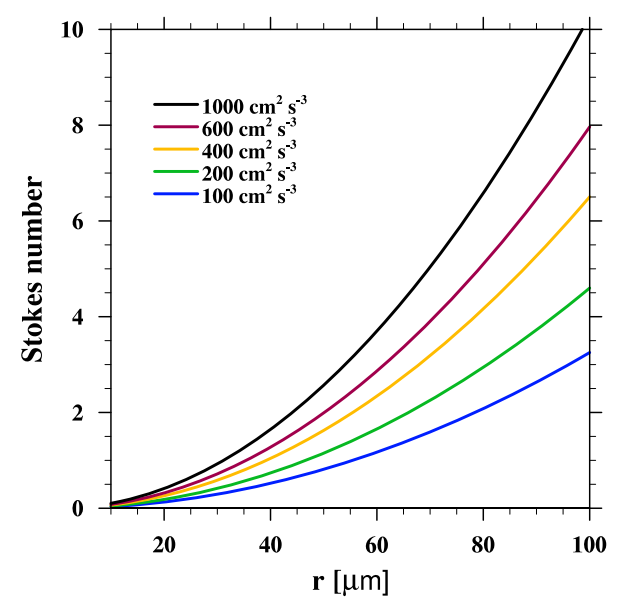

Figure 1. Stokes number against the particle radius for various energy dissipation rates.

$\left\langle\left|w_{\mathrm{r}}\right|\right\rangle=\left[\frac{2}{\pi}\left(w_{\text {shear }}^{2}+w_{\text {accel }}^{2}\right)\right]^{1 / 2}$,

$w_{\text {shear }}^{2}=\frac{R^{2} \epsilon}{15 v}$,

$w_{\mathrm{accel}}^{2}=\frac{1}{3} C_{w}\left(S t_{\max }\right) f_{\mathrm{KK}}$,

where $v$ is the kinematic viscosity and $C_{w}\left(S t_{\max }\right)=1+$ $0.6 \exp \left[-\left(S t_{\max }-1\right)^{1.5}\right]$. The formulation of $f_{\mathrm{KK}}$ was proposed by Kruis and Kusters (1997) as

$$
\begin{aligned}
f_{\mathrm{KK}} & =\frac{\gamma u_{\mathrm{rms}}^{2}}{\gamma-1}\left\{\left(\theta_{1}+\theta_{2}\right)-\frac{4 \theta_{1} \theta_{2}}{\left(\theta_{1}+\theta_{2}\right)}\left[\frac{1+\theta_{1}+\theta_{2}}{\left(1+\theta_{1}\right)\left(1+\theta_{2}\right)}\right]^{1 / 2}\right\} \\
& \times\left[\frac{1}{\left(1+\theta_{1}\right)\left(1+\theta_{2}\right)}-\frac{1}{\left(1+\gamma \theta_{1}\right)\left(1+\gamma \theta_{2}\right)}\right]
\end{aligned}
$$

where $\theta_{i}=\tau_{\mathrm{p}, i} / T_{\mathrm{L}}$ with $T_{\mathrm{L}}$ as the Lagrangian integral time and $\gamma=0.183 u_{\mathrm{rms}}^{2} /(\epsilon \nu)^{1 / 2}$. The Lagrangian integral time is parameterized as $T_{\mathrm{L}}=0.4 T_{\mathrm{e}}$, where $T_{\mathrm{e}}\left(=u_{\mathrm{rms}}^{2} / \epsilon\right)$ is the large-eddy turnover time (Kruis and Kusters, 1997; Zhou et al., 2001). In the equation, $\theta_{i}$ shows the relative particle relaxation time to the particle-flow interaction time. Note that this $\left\langle\left|w_{\mathrm{r}}\right|\right\rangle$ parameterization is for non-sedimenting droplets.

Onishi et al. (2009) concluded that gravitational sedimentation does not significantly influence turbulent collisions of cloud droplets. However, for this study, which extends the discussion to the small rain drop regime, the gravitational sedimentation cannot be ignored. Therefore, this study introduces a simple modification to make the model applicable to sedimenting droplets by considering the mechanism in which the gravitational settling shortens the interaction time of droplets with eddies (Onishi et al., 2009). Onishi et al. (2009) modeled the enlargement of the relative particle re- 
Table 1. Parameter values for $g_{11}$ model.

\begin{tabular}{lrrrrrrrr}
\hline & $A_{1}$ & $A_{2}$ & $a_{c}$ & $b_{c}$ & $a_{c 2}$ & $b_{c 2}$ & $a_{\alpha}$ & $b_{\alpha}$ \\
\hline Onishi et al. (2015) & 110 & 0.38 & 0.060 & 0.30 & - & - & 0.26 & 0.50 \\
Present & 110 & 0.32 & 0.046 & 0.36 & 0.094 & 0.25 & 0.23 & 0.50 \\
\hline
\end{tabular}

laxation time by gravity as

$\theta_{i, \text { sed }}=\sqrt{\frac{3\left(1-f\left(\theta_{i}\right)\right)+s_{\mathrm{v}}^{2}}{3\left(1-f\left(\theta_{i}\right)\right)}} \theta_{i}$,

where $f(\theta)$ is defined as the ratio of the particle velocity fluctuation to the flow velocity fluctuation, i.e., $f(\theta)=v_{\mathrm{p}}^{\prime 2} / u_{\mathrm{rms}}^{2}$, and $s_{\mathrm{V}}=V_{\mathrm{p}, \infty} / u_{\mathrm{rms}}$ is a non-dimensional parameter quantifying the influence of sedimentation. By replacing $\theta_{i}$ in Eq. (20) by $\theta_{i}$,sed, we obtain the radial relative velocity for droplets with gravitational sedimentation, $\left\langle\left|w_{\mathrm{r}}\right|\right\rangle_{\text {turb,sed }}$.

The above simple treatment is not yet complete. Ayala et al. (2008a) suggested the following two contributions of gravitational sedimentation on $\left\langle\left|w_{\mathrm{r}}\right|\right\rangle$ : (i) gravity reduces the interaction time of droplets with turbulent eddies, and therefore the variance of particle velocities is reduced, and (ii) gravity also decreases the correlation coefficient. The second contribution is missing in the present simple treatment. Nonetheless, since the present treatment leads to an improvement in the turbulent coagulation kernel, as shown in Sect. 4.3, this study adopts this simple treatment and leaves more robust treatment to future work.

The turbulent collision kernel formulated from the above $g_{12}$ and $\left\langle\left|w_{\mathrm{r}}\right|\right\rangle_{\text {turb,sed }}$ does not include the collision contribution due to the settling velocity difference. To include the contribution of the settling velocity difference, the following simple formulation was employed to obtain the total collision kernel.

$K_{\mathrm{c}, \text { total }}\left(r_{1}, r_{2}\right)=\left(K_{\mathrm{c}, \mathrm{turb}}^{2}\left(r_{1}, r_{2}\right)+K_{\mathrm{c}, \mathrm{grav}}^{2}\left(r_{1}, r_{2}\right)\right)^{1 / 2}$

Here, $K_{\mathrm{c} \text {,turb }}$ denotes the turbulent collision kernel obtained by $K_{\mathrm{c}, \text { turb }}=2 \pi R_{12}^{2}\left\langle\left|w_{\mathrm{r}}\right|\right\rangle_{\text {turb,sed }} g_{12}$.

\subsubsection{Turbulent enhancement on collision efficiency}

Onishi et al. (2015) employed the collision efficiency values of Pinsky et al. (2001) ( $E_{\mathrm{c}, \mathrm{PKS} 01}$ hereafter) and $\eta_{E}$ tabulated in Wang et al. (2008). These tabulated values spanned a relatively small range of particle sizes: the sizes of collector droplets $\left(r_{1}\right)$ were 20,30 , and $50 \mu \mathrm{m}$ and the size ratios $\left(r_{2} / r_{1}\right)$ were from 0.167 to 0.90 . Later, Wang and Grabowski (2009) tabulated the preliminary values of the enhancement factor for a wider range of droplet sizes: $r_{1}=20,30,40$, and $50 \mu \mathrm{m}$ and $r_{2} / r_{1}$ from 0.0 to 1.0. Note that the data for $r_{2} / r_{1}=0.0$ were simply set to the values for $r_{2} / r_{1}=0.0835$. It should also be noted that Wang and Grabowski (2009) tabulated the enhancement factors against the Hall collision ef- ficiency ( $E_{\mathrm{c}, \text { Hall }}$ hereafter; Hall, 1980). Unfortunately, inconsistencies exist between the two collision efficiency models. We found differences that are sometimes much larger than $10 \%$ of the mean between $E_{\mathrm{c}, \mathrm{PKS} 01}$ and $E_{\mathrm{c}, \mathrm{Hall}}$, particularly for small and large $r_{2} / r_{1}$ ratios, i.e., for $r_{2} / r_{1} \sim 0$ and $\sim 1$. These differences should be carefully compensated for in $\eta_{E}$. Wang and Grabowski (2009) tabulated the enhancement on $E_{\mathrm{c}, \text { Hall }}, \eta_{E}^{\# \text { Hall }}$. In fact, we observed an overestimation in turbulent enhancement on the autoconversion rate when we used $\eta_{E}^{\# \text { Hall }}$ for the SCE simulation with $E_{\mathrm{c}, \mathrm{PKS} 01}$. For Table 2, we calculated $\eta_{E}$ against $E_{\mathrm{c}, \mathrm{PKS} 01}\left(\eta_{E}^{\# \mathrm{PKS} 01}\right)$ from $\eta_{E}^{\# \text { Hall as }}$

$\eta_{E}^{\# \mathrm{PKS} 01}\left(r_{1}, r_{2}\right)=\frac{E_{\mathrm{c}, \mathrm{Hall}}\left(r_{1}, r_{2}\right)}{E_{\mathrm{c}, \mathrm{PKS} 01}\left(r_{1}, r_{2}\right)} \eta_{E}^{\# \text { Hall }}\left(r_{1}, r_{2}\right)$.

Following Wang and Grabowski (2009), this study simply sets the values for $r_{1} \leq 20 \mu \mathrm{m}$ to those at $r_{1}=20 \mu \mathrm{m}$, and similarly the values at $r_{1}=60 \mu \mathrm{m}$ to those at $r_{1}=50 \mu \mathrm{m}$. The factor is set to unity for $r_{1}=100 \mu \mathrm{m}$ and larger. Also, following Seifert et al. (2010), for $100 \leq \epsilon \leq 600 \mathrm{~cm}^{2} \mathrm{~s}^{-3}$, this study linearly interpolates/extrapolates between the values of $\eta_{E}^{\# P K S 01}$ at $\epsilon=100 \mathrm{~cm}^{2} \mathrm{~s}^{-3}$ and at $\epsilon=400 \mathrm{~cm}^{2} \mathrm{~s}^{-3}$. For $\epsilon>600 \mathrm{~cm}^{2} \mathrm{~s}^{-3}$ the extrapolated values at $\epsilon=600 \mathrm{~cm}^{2} \mathrm{~s}^{-3}$ are used for $\eta_{E}^{\# \mathrm{PKS} 01}$.

\section{Direct numerical simulations}

\subsection{Computational methods}

We now solve the three-dimensional continuity and NavierStokes equations for incompressible flows:

$\nabla \cdot \mathbf{U}=0$

$\frac{\partial \mathbf{U}}{\partial t}+(\mathbf{U} \cdot \nabla) \mathbf{U}=-\frac{1}{\rho} \nabla p+v \nabla^{2} \mathbf{U}+\mathbf{F}(\boldsymbol{x}, t)$.

The kinematic viscosity $v$ is set to $1.5 \times 10^{-5} \mathrm{~m}^{2} \mathrm{~s}^{-3}$, which is the value for atmospheric air at $1 \mathrm{~atm}$ and $298 \mathrm{~K}$. The last term on the right-hand side represents the external forcing needed to achieve a statistically steady state. This study employs reduced-communication forcing (Onishi et al., 2011), which is suitable for massively parallel finite-difference models, to maintain the kinetic energy with $|\boldsymbol{k}|<2.5$, where $\boldsymbol{k}$ is a wavevector. Spatial derivatives are calculated using fourth-order central differences. The conservative scheme of Morinishi et al. (1998) is employed for the advection term, 
Table 2. Enhancement factor for the Pinsky collision efficiency (PKS01), $\eta_{E}^{\# \mathrm{PKS} 01}$, for (a) $\epsilon=100 \mathrm{~cm}^{2} \mathrm{~s}^{-3}$ and (b) $\epsilon=$ $400 \mathrm{~cm}^{2} \mathrm{~s}^{-3}$.

\begin{tabular}{rrrrr}
\hline (a) & $r_{1}=$ & & & \\
$r_{2} / r_{1}$ & $20 \mu \mathrm{m}$ & $30 \mu \mathrm{m}$ & $40 \mu \mathrm{m}$ & $50 \mu \mathrm{m}$ \\
\hline 0.0 & 1.74 & 1.77 & 1.49 & 1.21 \\
0.1 & 5.26 & 3.55 & 2.31 & 1.65 \\
0.2 & 2.67 & 0.742 & 1.29 & 1.04 \\
0.3 & 1.75 & 0.733 & 1.15 & 1.04 \\
0.4 & 0.995 & 0.953 & 1.11 & 1.06 \\
0.5 & 0.955 & 1.06 & 1.03 & 1.03 \\
0.6 & 0.730 & 1.11 & 1.00 & 1.03 \\
0.7 & 0.701 & 1.07 & 0.983 & 0.991 \\
0.8 & 1.01 & 1.18 & 1.06 & 1.01 \\
0.9 & 1.63 & 1.81 & 1.34 & 1.31 \\
1.0 & 29.2 & 6.10 & 2.89 & 3.14 \\
\hline $\mathbf{b})$ & $r_{1}=$ & & & \\
$r_{2} / r_{1}$ & $20 \mu \mathrm{m}$ & $30 \mu \mathrm{m}$ & $40 \mu \mathrm{m}$ & $50 \mu \mathrm{m}$ \\
\hline 0.0 & 4.98 & 3.59 & 2.52 & 1.45 \\
0.1 & 10.7 & 5.45 & 3.13 & 1.86 \\
0.2 & 4.03 & 0.879 & 1.51 & 1.20 \\
0.3 & 2.08 & 0.758 & 1.22 & 1.15 \\
0.4 & 1.05 & 0.973 & 1.14 & 1.10 \\
0.5 & 0.751 & 1.19 & 1.10 & 1.05 \\
0.6 & 0.832 & 1.29 & 1.10 & 1.07 \\
0.7 & 0.929 & 1.29 & 1.10 & 1.02 \\
0.8 & 1.42 & 1.41 & 1.21 & 1.09 \\
0.9 & 3.94 & 2.19 & 1.51 & 1.34 \\
1.0 & 22.6 & 5.47 & 2.18 & 1.88 \\
\hline & & & &
\end{tabular}

and the second-order Runge-Kutta scheme is employed for time integration. To solve the velocity-pressure coupling, we use the highly simplified marker and cell (HSMAC) scheme (Hirt and Cook, 1972), which iterates until the rms of the velocity divergence becomes smaller than $\delta / \Delta$, where $\Delta$ is the grid spacing and $\delta$ is chosen to be $10^{-3}$. The governing equations are discretized by using a cubic domain of length $2 \pi L_{0}$, where $L_{0}$ is the representative length. Periodic boundary conditions are applied in all three directions. The flow cube is discretized uniformly into $N^{3}$ grid points, resulting in $\Delta=2 \pi L_{0} / N$.

Under the limit of a large ratio of the density of the particle material to that of the fluid $\left(\rho_{\mathrm{p}} / \rho_{\mathrm{f}} \gg 1\right)$, the governing equation for water droplets is given by

$\frac{\mathrm{d} \mathbf{V}}{\mathrm{d} t}=-\frac{f}{\tau_{\mathrm{p}}}(\mathbf{V}-(\mathbf{U}(\boldsymbol{x}, t)+\boldsymbol{u}(\boldsymbol{x}, t)))+\mathbf{F}_{\text {impulse }}+\boldsymbol{g}$,

where $\mathbf{V}$ is the particle velocity, $\mathbf{U}$ is the air velocity at the position of the droplet, $\boldsymbol{u}$ is the disturbance flow velocity due to the surrounding droplets, and $\tau_{\mathrm{p}}$ is the particle relaxation time defined as $\tau_{\mathrm{p}}=(2 / 9)\left(\rho_{\mathrm{p}} / \rho_{\mathrm{f}}\right)\left(r^{2} / v\right)$, in which $r$ is the particle radius. $\mathbf{F}_{\text {impulse denotes the impulsive force due to }}$ collisions and $\mathbf{g}$ is the gravity vector $(=(-g, 0,0)$, where $g$ is the gravitational acceleration). The ratio of the density of the particle material to that of the fluid, $\rho_{\mathrm{p}} / \rho_{\mathrm{f}}$, is set to $8.43 \times$ $10^{2}$ at $1 \mathrm{~atm}$ and $298 \mathrm{~K}$, and $f$ is the drag coefficient defined as the ratio between the nonlinear drag and the linear drag (Rowe and Henwood, 1961). It should be noted that Eq. (26), which adopts the point-particle assumption, is inaccurate for large $S t$ particles whose radii are not small enough compared to the Kolmogorov scale.

The second-order Runge-Kutta method is used for the time integration. The flow velocity at the droplet position $\mathbf{U}$ is linearly interpolated from the adjacent grid values. This simple linear interpolation is justified through comparisons with the cubic Hermitian, cubic Lagrangian, and fifth-order Lagrangian interpolations from Sundaram and Collins (1996). The disturbance flow $\boldsymbol{u}$, which denotes the hydrodynamic interaction, is calculated by using the BiSM (Onishi et al., 2013). The particle mass and volume fractions are so dilute that the flow modulation is ignored.

\subsection{Computation for turbulent collision statistics}

After the background airflow has reached a statistically stationary state, monodispersed water droplets are introduced into the flow. After a period exceeding 3 times the eddyturnover time $T_{0}=L_{0} / U_{0}$, collision detection is then started. Droplets are allowed to overlap (ghost-particle condition) and a collision is judged from the trajectories of a pair of droplets by assuming linear particle movement for the time interval $\Delta t$.

The detailed description of the procedures for calculating collision statistics can be found in Onishi et al. (2013), who conducted the DNS for $R e_{\lambda}$ up to 530. This study performed additional simulations to push the maximum $R e_{\lambda}$ forward, up to 1140 . The computational settings for the present simulations are summarized in Table 3.

\subsection{Computation for size evolutions due to collisional growth}

To obtain reference data regarding droplet collisional growth, we tracked the growth of droplets that initially had the following exponential size distribution (e.g., Soong, 1974):

$f_{0}(x)=\frac{n_{0}}{x_{\mathrm{m} 0}} \exp \left(-x / x_{\mathrm{m} 0}\right)$,

where $x_{\mathrm{m} 0}$ is the mass of a droplet with a radius of $r_{\mathrm{m} 0}$ and $n_{0}$ is the initial number density. We carried out two cases: one with $r_{\mathrm{m} 0}=15 \mu \mathrm{m}$ and $n_{0}=1.42 \times 10^{8} \mathrm{~m}^{-3}$ and the other with $r_{\mathrm{m} 0}=10 \mu \mathrm{m}$ and $n_{0}=4.79 \times 10^{8} \mathrm{~m}^{-3}$. The corresponding initial liquid water content was $2.0 \mathrm{~g} \mathrm{~m}^{-3}$ for both cases. It was assumed that colliding particles immediately united without breakups and conserved mass and momentum.

Table 4 summarizes the computational parameters for the flow calculation as well as the obtained flow statistics for the 
Table 3. Case configurations and typical turbulence statistics. $R e=U_{0} L_{0} / v, u^{\prime}$ is the rms of flow velocity fluctuation, $k_{\max }(=N / 2)$ is the maximum wavenumber, $l_{\eta}$ is the Kolmogorov scale, and $R e_{\lambda}$ is the Taylor-microscale-based Reynolds number. $N_{\mathrm{p}}$ is the total number of particles.

\begin{tabular}{rrrr|rrr|r}
\hline & $N^{3}$ & $L_{0}[\mathrm{~m}]$ & $R e$ & $u^{\prime}$ & $k_{\max } l_{\eta}$ & $R e_{\lambda}$ & $N_{\mathrm{p}}$ \\
\hline $\mathrm{N} 4000$ & $4000^{3}$ & 0.312 & 14100 & 1.01 & 2.10 & 874 & $1.60 \times 10^{9}$ \\
$\mathrm{~N} 6000$ & $6000^{3}$ & 0.468 & 24200 & 1.01 & 2.11 & 1140 & $5.40 \times 10^{9}$ \\
\hline
\end{tabular}

Table 4. Case configurations and typical turbulence statistics. $R e=U_{0} L_{0} / \nu$, where $U_{0}$ is the representative velocity and $L_{0}$ is the representative length, $u^{\prime}$ is the rms of the flow velocity fluctuation, $k_{\max }(=N / 2)$ is the maximum wavenumber, $l_{\eta}$ is the Kolmogorov scale, $\lambda$ is the local shear rate, and $R e_{\lambda}$ is the Taylor-microscale-based Reynolds number.

\begin{tabular}{lrrr|rrrr}
\hline & $N^{3}$ & $L_{0}[\mathrm{~m}]$ & $R e$ & $u^{\prime}$ & $k_{\max } l_{\eta}$ & $R e_{\lambda}$ & $\epsilon\left[\mathrm{cm}^{3} \mathrm{~s}^{-2}\right]$ \\
\hline NoT & $32^{3}$ & 0.0127 & 0 & 0 & - & 0 & 0 \\
\hline T100 & $96^{3}$ & 0.0180 & 97.4 & 1.00 & 2.04 & 66.1 & 100 \\
T & $96^{3}$ & 0.0127 & 97.4 & 1.00 & 2.04 & 66.1 & 400 \\
T1000 & $96^{3}$ & 0.0101 & 97.4 & 1.00 & 2.04 & 66.1 & 1000 \\
TR127 & $256^{3}$ & 0.0338 & 360 & 0.98 & 2.06 & 127 & 400 \\
TR206 & $512^{3}$ & 0.0669 & 908 & 1.00 & 2.06 & 206 & 400 \\
TR333 & $1000^{3}$ & 0.135 & 2220 & 1.00 & 2.07 & 333 & 400 \\
\hline
\end{tabular}

collision growth simulations. In cases T100, T, and T1000, the same grid configuration with the same Reynolds number was calculated, but the energy dissipation rates, which are in the typical range observed in turbulent atmospheric clouds, were 100,400 , and $1000 \mathrm{~cm}^{3} \mathrm{~s}^{-2}$, respectively. Cases T, TR127, TR206, and TR333 obtained flows with the same energy dissipation rate $\left(400 \mathrm{~cm}^{3} \mathrm{~s}^{-2}\right)$ but with different $R e_{\lambda}$ values. Onishi et al. (2015) have already presented these cases, except for TR333 with $r_{\mathrm{m} 0}=15 \mu \mathrm{m}$. The present study additionally performed the case TR 333 with $r_{\mathrm{m} 0}=15 \mu \mathrm{m}$ to obtain a clear Reynolds-number dependence, as well as cases T, TR127, and TR206 with $r_{\mathrm{m} 0}=10 \mu \mathrm{m}$.

\section{Results and discussion}

\subsection{Estimate for Reynolds-number dependence of clustering effect of small-St particles}

Onishi et al. (2013) observed that the clustering effect and consequently the collision kernel decreases as the Reynolds number increases for $R e_{\lambda}>100$ and $S t=0.4$. Later, Onishi and Vassilicos (2014) clarified that the Reynolds-number dependence of $g_{11}$ observed for $1 / 3<S t<1$ is due to internal intermittency of the three-dimensional turbulence.

To quantify the influence of intermittence on $g_{11}$, we need to separate the local quantity from the global (average) quantity. Kolmogorov (1962) introduced the local energy dissipation as

$\epsilon_{l}(\boldsymbol{x}, t)=\frac{3}{4 \pi l^{3}} \int_{|\boldsymbol{y}| \leq l} \epsilon^{\#}(\boldsymbol{x}+\boldsymbol{y}, t) \mathrm{d} \boldsymbol{y}$, where superscript \# denotes the local quantity. It was supposed that the probability density function (PDF) of $\epsilon_{l}$ follows a log-normal distribution if $l$ is much smaller than the flow integral scale. Assuming $l \sim \eta$, we obtain

$$
P_{\mathrm{LN}}\left(\epsilon^{*} \mid \mu, \sigma^{2}\right)=\frac{1}{\sqrt{2 \pi} \sigma \epsilon^{*}} \exp \left(\frac{-\left(\ln \epsilon^{*}-\mu\right)^{2}}{2 \sigma^{2}}\right),
$$

where $\epsilon^{*}=\epsilon_{\eta}$. Parameters $\sigma$ and $\mu$ appear in the first and second moments of $\epsilon^{*}$ as

$\left\langle\epsilon^{*}\right\rangle(=\epsilon)=\exp \left(\mu+\sigma^{2} / 2\right)$

and

$\left\langle\epsilon^{* 2}\right\rangle=\exp \left(2 \mu+2 \sigma^{2}\right)$,

respectively.

The intermittency is measured by the flatness factor $F$, defined as

$F=\frac{\left\langle\left(\partial u_{1} / \partial x_{1}\right)^{4}\right\rangle}{\left\langle\left(\partial u_{1} / \partial x_{1}\right)^{2}\right\rangle^{2}}$

It is observed that $F$ follows a power law relation with $R e_{\lambda}$, for example $F \sim R e_{\lambda}^{3 / 8}$ (Pope, 2000). Given $\partial u_{1} / \partial x_{1} \sim$ $\left(\epsilon_{\eta} / \nu\right)^{1 / 2}=\left(\epsilon^{*} / \nu\right)^{1 / 2}$, we obtain

$F \sim \frac{\left\langle\epsilon^{* 2}\right\rangle}{\epsilon^{2}} \sim R e_{\lambda}^{3 / 8}$.

Substitution of Eqs. (30) and (31) into Eq. (33) yields

$\sigma^{2}=\frac{3}{8} \ln \left(R e_{\lambda}\right)$. 
Eq. (30) then yields

$\mu=\ln \epsilon e_{\lambda}^{-3 / 16}$.

That is, $P_{\mathrm{LN}}\left(\epsilon^{*} \mid \mu, \sigma^{2}\right)$ can be rewritten as $P_{\mathrm{LN}}\left(\epsilon^{*} \mid R e_{\lambda}\right)$. We can define a local $S t, S t^{*}$, as

$S t^{*}=S t \times\left(\frac{\epsilon^{*}}{\epsilon}\right)^{1 / 2}$,

the PDF of which follows

$P\left(S t^{*} \mid S t, R e_{\lambda}\right)=\frac{2 \epsilon S t^{*}}{S t^{2}} P_{\mathrm{LN}}\left(\epsilon\left(\frac{S t^{*}}{S t}\right)^{2} \mid R e_{\lambda}\right)$.

It should be emphasized that the shape of $P_{\mathrm{LN}}$ (and consequently $P$ ) depends on $R e_{\lambda}$. If we assume a universal radial distribution function at contact separation against $S t^{*}-$ $g_{11}^{\# \text { \#univ }}\left(S t^{*}\right)-$, the global clustering effect can be obtained as

$g_{11}\left(S t, R e_{\lambda}\right)=\int_{0}^{\infty} g_{11}^{\# \text { univ }}\left(S t^{*}\right) P\left(S t^{*} \mid S t, R e_{\lambda}\right) \mathrm{d} S t^{*}$.

It should be noted that $g_{11}$ depends on $R e_{\lambda}$, whereas $g_{11}^{\# \text { \#niv }}$ does not (which is why it is called universal). For $S t^{*} \ll 1$, the universal clustering effect would have the form $g_{11}^{\text {\#univ }}=$ $A_{1} S t^{* 2}+1$ by following Eq. (10). Substitution of this form into Eq. (38) yields $g_{11}\left(S t \ll 1, R e_{\lambda}\right)=A_{1} S t^{2}+1$, regardless of the value of $R e_{\lambda}$. This explains why the $g_{11}$ for $S t=0.1$ does not show a significant Reynolds-number dependence. For a moderate $S t^{*}$, we simply formulate the universal function by following Eqs. (10) and (11) but without the smoothing operators, as follows:

$$
\begin{aligned}
g_{11}^{\# \text { univ }}\left(S t^{*}\right) & =H\left(S t^{*}-S t_{a}^{*}\right) A_{1}^{*} S t^{* 2} \\
& +H\left(S t_{a}^{*}-S t^{*}\right) A_{2}^{*} S t^{*-2},
\end{aligned}
$$

where $A_{1}^{*}$ and $A_{2}^{*}$ are empirical parameters and $S t_{a}^{*}$ is defined as $\left(A_{2}^{*} / A_{1}^{*}\right)^{1 / 4}$. Based on the DNS data for $S t=0.1,0.4$, and 0.6 in the flow with $R e_{\lambda}=130$, we found that $A_{1}^{*}=110$ and $A_{2}^{*}=0.073$ work reasonably well. Although we have no justification for this universal function, it can provide $g_{11}$ for arbitrary $S t(<1)$ and $R e_{\lambda}$ through Eq. (38). As we cannot analytically calculate the integration in Eq. (38), we have to numerically calculate it to obtain $g_{11}$ for a certain combination of $S t$ and $R e_{\lambda}$. We calculated $g_{11}$ for $S t=0.1,0.4$, and 0.6 with $R e_{\lambda}=100,200,400,1000,4000$, and 10000 . We then obtained the following empirical formulations by applying the least-squares method to the calculated results.

$$
\begin{aligned}
& g_{11}\left(S t=0.1, R e_{\lambda}\right) \sim 2.1, \\
& g_{11}\left(S t=0.4, R e_{\lambda}\right) \sim 19.3-1.9 \log _{10} R e_{\lambda}, \\
& g_{11}\left(S t=0.6, R e_{\lambda}\right) \sim 34.3-3.9 \log _{10} R e_{\lambda} .
\end{aligned}
$$

Figure 2 shows a comparison between $g_{11}$ values from the above equations and those from the DNS. The figure shows

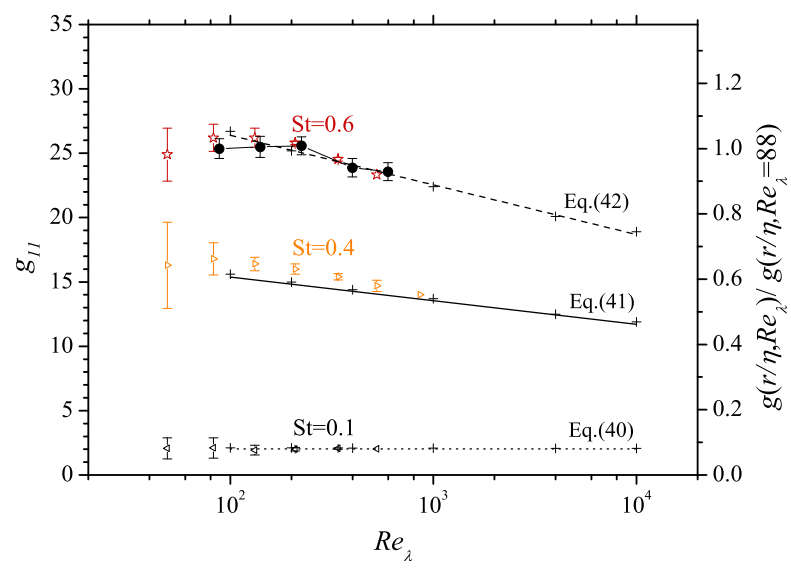

Figure 2. Radial distribution function (RDF) at the contact of monodisperse particles with $S t=0.1,0.4$, and 0.6 against $R e_{\lambda}$. The plotted open symbols are the reference DNS results. The lines are the results of Eqs. (40), (41), and (42), which were fitted to the sample values $(+)$ with using the least-squares method. The error bars show \pm one standard deviation obtained from more than three runs, with each run lasting for a time $T_{0}=L_{0} / U_{0}$. Black solid circle (right $y$ axis) denotes the RDF at $r / \eta=0.25$ for $S t=0.6$, normalized by the value at $R e_{\lambda}=88$ reported in Ireland et al. (2016a).

that the empirical estimates can reproduce the Reynoldsnumber dependence of $g_{11}$ correctly. The figure includes the data for $S t=0.6$ recently reported in Ireland et al. (2016a). The two DNS datasets for $S t=0.6$ agree well. Ireland et al. (2016a) argue that the Reynolds-number dependence is weak for the Reynolds number range explored by DNS, but the argument should be carefully examined when extrapolating to atmospheric flows. Figure 2 shows indeed a weak decrease in $g_{11}$ for Stokes numbers between 0.3 and 1 .

\subsection{Modeling of clustering effect}

Figure 3 shows a comparison between direct numerical simulation results and model predictions for $g_{11}$. The dashed lines are the prediction by the Onishi model (Onishi et al., 2015), and the solid lines are the predictions by the present updated model. The DNS data for $S t \leq 1$ and for $R e_{\lambda} \leq 530$ were obtained from the table in Onishi et al. (2015). The data for $S t=1.4,2,4$, and 8 were newly obtained. The results for $R e_{\lambda}=874$ and 1140 (these Reynolds numbers are the largest ever achieved for turbulent particle collision statistics) are included in the figure. The DNS data show a decreasing trend for $S t<1$ for the moderate Reynolds number range of $100 \lesssim R e_{\lambda} \lesssim 1000$. This decreasing trend with respect to $R e_{\lambda}$ is attributed to the flow intermittency (Onishi and Vassilicos, 2014) as discussed in the previous subsection. The black solid line is the estimated $g_{11}$ for $S t=0.4$ and the black dashed line is for $S t=0.6$ (Eqs. 41 and 42, respectively). The present Onishi model shows slightly better agreement with the DNS data in terms of the slopes in comparison with the 


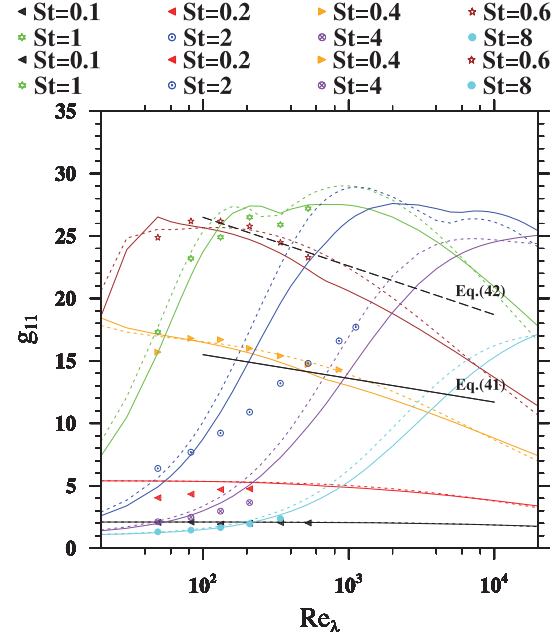

Figure 3. Radial distribution function at the contact of monodisperse particles, $g_{11}$, against $R e_{\lambda}$. The plotted points are the reference DNS results, the dotted lines are the prediction with the coefficients of Onishi et al. (2015), and the solid lines are the present prediction.

original model. For $S t>1$, the DNS data show increasing trends for the moderate $R e_{\lambda}$ range, and those trends are predicted by the present parameterization, although the rate for $S t=2$ is overestimated. One significant feature of the Onishi $g_{11}$ model is that maximum clustering occurs at a larger $S t$ for a larger $R e_{\lambda}$. This shows a clear contrast with the AyalaWang model, which was designed to show maximum clustering at $S t \sim 1$ regardless of $R e_{\lambda}$.

The updated parameterization leads to improvement, particularly for the $S t \geq 1$ regime. For example, in the case of $R e_{\lambda}=127$, the rms values of the relative errors of the prediction with the original parameters for (i) $S t=0.1,0.2,0.4$, and 0.6 and for (ii) $S t=1,1.4,2,4$, and 8 were (i) 0.081 and (ii) 0.239 . The rms values with the present parameters were (i) 0.075 and (ii) 0.113 .

\subsection{Turbulent coagulation kernels for small Reynolds-number flow}

Figure 4 shows a comparison between model predictions and DNS results of the coagulation kernel $K_{\text {coag }}\left(r_{1}, r_{2}\right)$ for $r_{1}=$ $30 \mu \mathrm{m}, R e_{\lambda}=127$, and $\epsilon=400 \mathrm{~cm}^{2} \mathrm{~s}^{-3}$. The kernel is normalized by the collision radius $R$ and the local velocity gradient $\lambda\left(=(\epsilon / \nu)^{1 / 2}\right)$. The reference DNS considers the hydrodynamic interaction and the gravitational droplet sedimentations. We observe a large discrepancy for $r_{2} \sim 30 \mu \mathrm{m}\left(=r_{1}\right)$, where the turbulence enhancement on collision efficiency is difficult to define, because the collision efficiency for $r_{1}=r_{2}$ cannot be defined for stagnant flow. Otherwise, the model predictions (Ayala-Wang model and Onishi model) agree well with the DNS results. As an example, we also observe a slight improvement in the Onishi model by including the

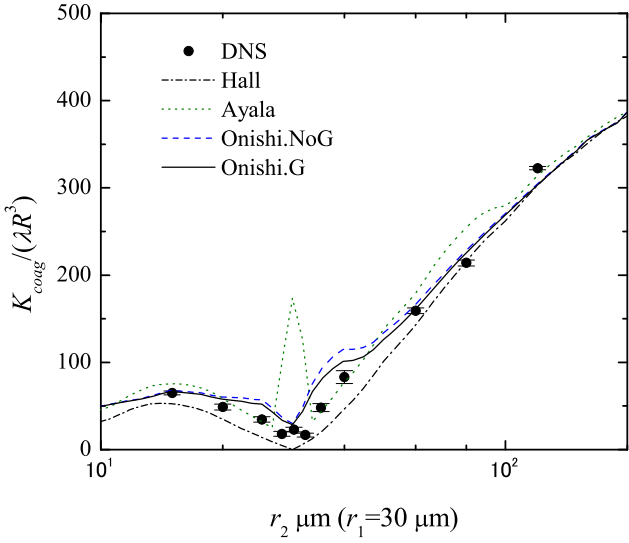

Figure 4. Non-dimensionalized coagulation kernels for $r_{1}=30 \mu \mathrm{m}$ in the turbulent flow with $R e_{\lambda}=127$ and $\epsilon=400 \mathrm{~cm}^{2} \mathrm{~s}^{-3}$. The error bars show \pm 1 standard deviation obtained from more than three runs, with each run lasting for a time $T_{0}=L_{0} / U_{0}$.

sedimentation effect on $\left\langle\left|w_{\mathrm{r}}\right|\right\rangle$ (Sect. 2.3.2) on the data for $r_{2}=40 \mu \mathrm{m}$.

The Ayala-Wang model shows a local maximum around $r_{2}=r_{1}$. The DNS results also show a convex shape, but the value at $r_{2}=r_{1}$ is much smaller than the prediction by the Ayala-Wang model. In contrast, the Onishi model does not show such a local maximum at $r_{2}=r_{1}$ but does provide values much closer to DNS elsewhere. The convex shape is related to the diffusion effect denoted by $r_{\mathrm{d}}$ in Eq. (7). Equation (16) for $g_{12}$, employed in the Onishi model, was formulated for non-sedimenting droplets and this equation therefore leads to weaker acceleration-driven diffusion, i.e., smaller $r_{\mathrm{d}}$ (Ayala et al., 2008a). This can explain why the Onishi model does not show the convex shape.

Figure 5 shows the ratio of the turbulent coagulation kernel to the Hall kernel for the turbulent flow with $R e_{\lambda}=127$ and $\epsilon=400 \mathrm{~cm}^{2} \mathrm{~s}^{-3}$. The level of the ratio is basically similar for both the Ayala-Wang and Onishi models, and the ratio is nearly unity when the droplets are above $100 \mu \mathrm{m}$.

\subsection{Reynolds-number dependence of kernel models}

Figure 6 shows the ratio of the coagulation kernel for $R e_{\lambda}=$ $10^{4}$ to that for $R e_{\lambda}=10^{3}$. It should be noted that the $E_{\mathrm{c}}$ and $\eta_{E}$ models employed in the Ayala-Wang and Onishi kernels do not consider the Reynolds dependence. Therefore, the figure actually shows the ratio of the geometric collision kernels, i.e., the ratio of $\left\langle\left|w_{\mathrm{r}}\right|\right\rangle g_{12}$. The Ayala-Wang kernel increases for the autoconversion region $\left(r_{1}, r_{2}<40 \mu \mathrm{m}\right)$ and the accretion region $\left(r_{1}<40\right.$ and $r_{2}>40$, and $r_{1}>40$ and $\left.r_{2}<40 \mu \mathrm{m}\right)$. The Onishi kernel decreases for the corresponding autoconversion region, but increases for the rainrain self-collection region $\left(r_{1}, r_{2}>40 \mu \mathrm{m}\right)$.

Figure 7 shows the ratio of $g_{12}$ for $R e_{\lambda}=10^{4}$ to that for $R e_{\lambda}=10^{3}$. It should be noted that the form of 

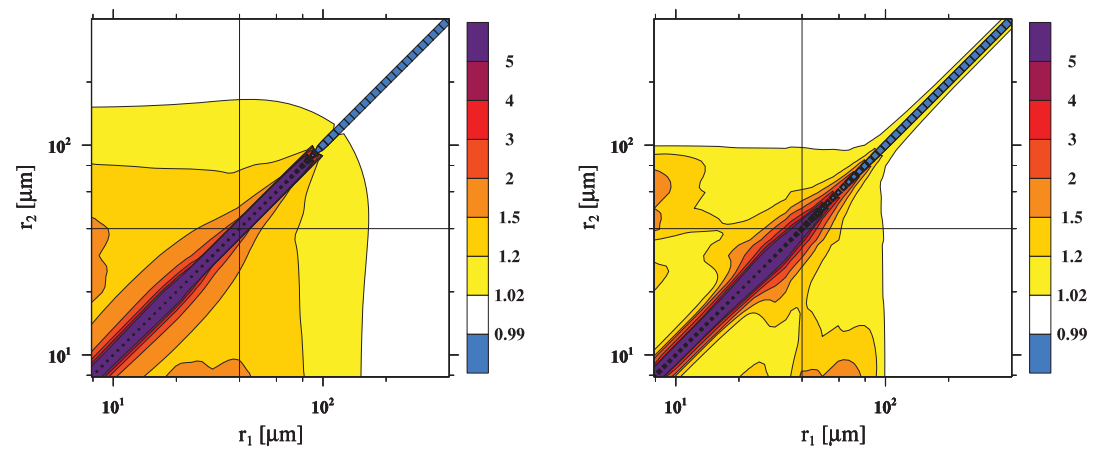

Figure 5. Ratio of the turbulent coagulation kernel to the Hall kernel in the turbulent flow with $\operatorname{Re}_{\lambda}=127$ and $\epsilon=400 \mathrm{~cm}^{2} \mathrm{~s}-3$. (a) AyalaWang kernel and (b) the present Onishi kernel.
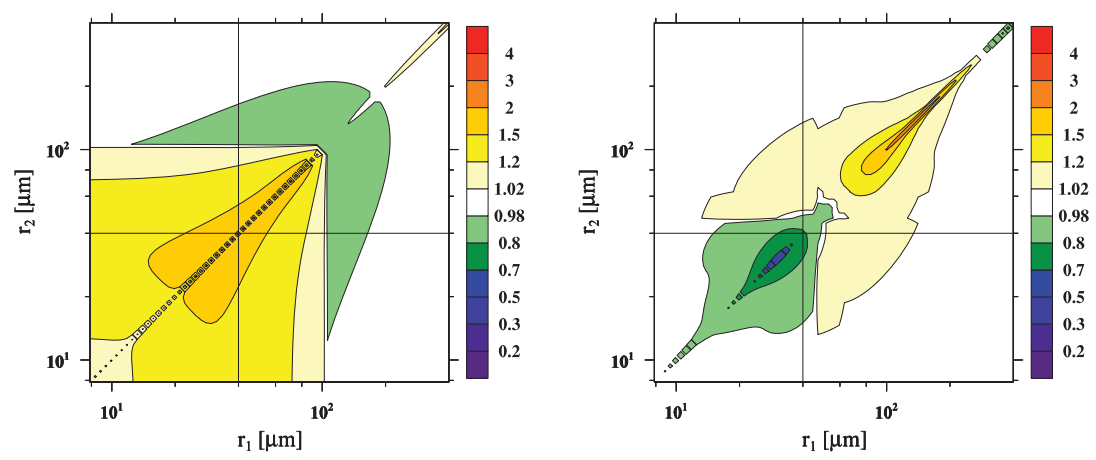

Figure 6. Ratio of the coagulation kernel for $R e_{\lambda}=10^{4}$ to that for $R e_{\lambda}=10^{3}$. (a) Ayala-Wang kernel and (b) the present Onishi kernel.

Eq. (22) violates the spherical form and we cannot rigorously define $g_{12 \text {,total }}$ and $\left\langle\left|w_{\mathrm{r}}\right|\right\rangle_{\text {total }}$ that formulate $K_{\mathrm{c}, \text { total }}=$ $2 \pi R_{12}^{2}\left\langle\left|w_{\mathrm{r}}\right|\right\rangle_{\text {total }} g_{12, \text { total. }}$. Here, we simply considered $g_{12}$ expressed by Eq. (12) as the $g_{12, \text { total }}$ for the total kernel and obtained $\left\langle\left|w_{\mathrm{r}}\right|\right\rangle_{\text {total }}=K_{\mathrm{c}, \text { total }} /\left(2 \pi R_{12}^{2} g_{12}\right)$. As designed, the Ayala-Wang kernel shows the increase for increasing $R e_{\lambda}$ for both the autoconversion and the accretion regions. In contrast, the Onishi kernel shows a decrease for the autoconversion region, but a significant increase for the accretion region and the rain-rain self-collection region (i.e., $r_{1}, r_{2}>40 \mu \mathrm{m}$ ). This is due to the shift of the maximum clustering toward larger $S t$ with increasing $R e_{\lambda}$.

Figure 8 shows the ratio of the radial relative velocity for $R e_{\lambda}=10^{4}$ to that for $R e_{\lambda}=10^{3}$. The Ayala-Wang kernel shows little Reynolds-number dependence. In contrast, the Onishi kernel shows significant Reynolds-number dependence, which tends to be opposite to the Reynolds-number dependence of $g_{12}$ and thus weakens the Reynolds-number dependence of the collision kernel.

The Reynolds-number dependence of the clustering effect is larger than that of the radial relative velocity, and the contour shape of Fig. 6 is more similar to Fig. 7 than to Fig. 8 for both the Ayala-Wang and the Onishi kernels. That is, the Reynolds-number dependence of the two kernels can mostly be attributed to the $g_{12}$ parameterizations.
Note that the Fortran 90 code used to calculate the present Onishi kernel is provided in the Supplement.

\subsection{Turbulence enhancement of autoconversion rate}

We investigated the turbulence enhancement on the autoconversion rate, which is the conversion rate from the cloud category $(r<40 \mu \mathrm{m})$ to the rain category due to collisions between the small cloud droplets. The Ayala-Wang kernel and the present Onishi kernel were employed to calculate the coagulation growth of droplets modeled by the stochastic collision-coalescence equation (SCE):

$$
\begin{aligned}
\frac{\partial n_{\mathrm{f}}(m, t)}{\partial t} & =\int_{0}^{m / 2} K_{\text {coag }}\left(m-m^{\prime}, m^{\prime}\right) n_{\mathrm{f}}\left(m-m^{\prime}, t\right) n_{\mathrm{f}}(m, t) \mathrm{d} m^{\prime} \\
& -\int_{0}^{\infty} K_{\text {coag }}\left(m, m^{\prime}\right) n_{\mathrm{f}}(m, t) n_{\mathrm{f}}\left(m^{\prime}, t\right) \mathrm{d} m^{\prime},
\end{aligned}
$$

where $m$ is the particle mass and $n_{\mathrm{f}}$ is the number density function. The coagulation component of the spectral bin model in the Multi-Scale Simulator for the Geoenvironment (MSSG-Bin) cloud physics model (Onishi and Takahashi, 2012) was used to solve the SCE. The mass coordinate $m$ was discretized as $m_{k}=2^{1 / s} m_{k-1}$, where $s$ was set to 16 . 

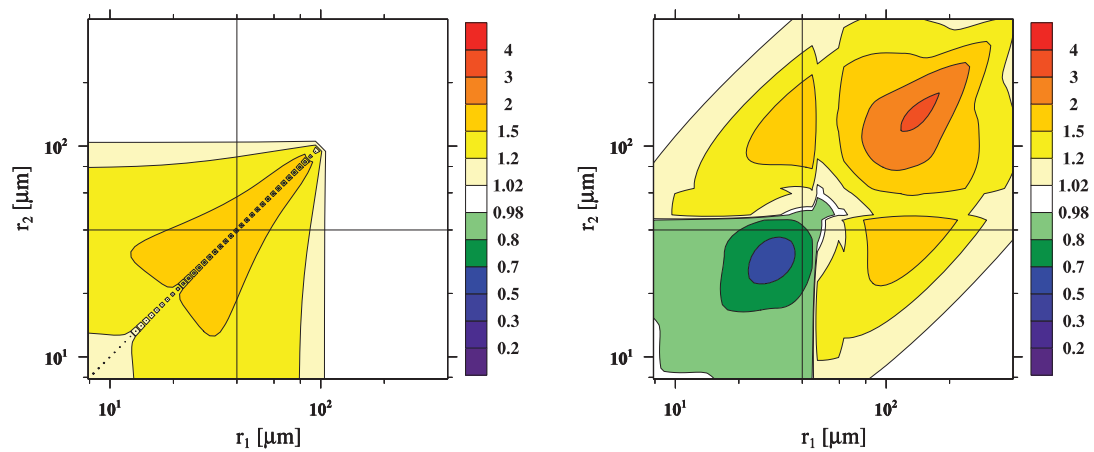

Figure 7. Ratio of the clustering effect $g_{12}$ for $R e_{\lambda}=10^{4}$ to that for $R e_{\lambda}=10^{3}$.
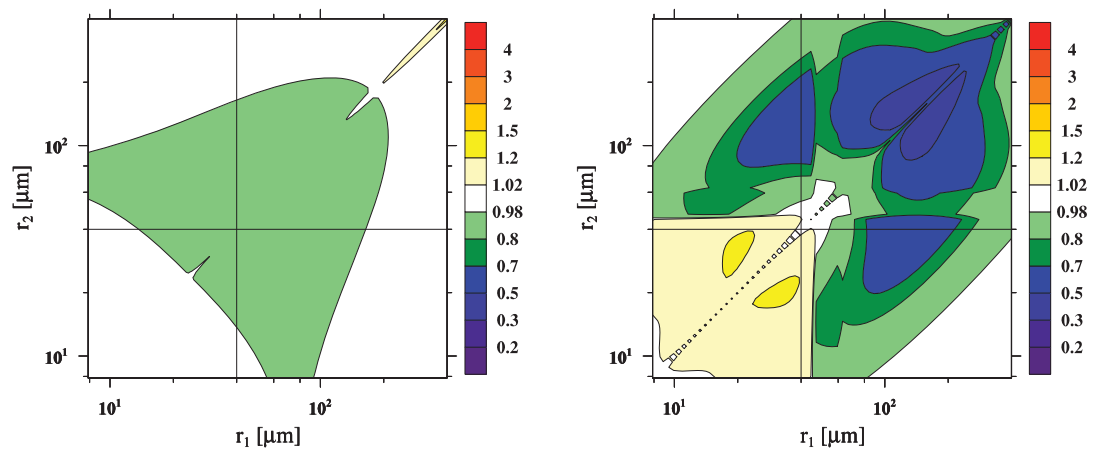

Figure 8. Ratio of the radial relative velocity at contact separation $\left\langle\left|w_{\mathbf{r}}\right|\right\rangle$ for $R e_{\lambda}=10^{4}$ to that for $R e_{\lambda}=10^{3}$.

The representative radius of the first bin was $2.7 \mu \mathrm{m}$ and 528 classes were calculated, the largest class of which had a representative radius of $5.4 \mathrm{~mm}$. The SCE solution is basically a mean-field approximation. In contrast, the LCS acts as a reference model as it includes all turbulence effects directly in its Lagrangian particle simulation. Due to the high computational cost, however, the LCS is restricted to moderate Reynolds number (here up to $R e_{\lambda}=333$ ).

Following Seifert et al. (2010), Onishi et al. (2015) used a quantitative measure of the turbulence enhancement focusing on the timescale of the autoconversion process. The time required for a cloud to convert $10 \%$ of its cloud mass into rain category drops is expressed as $t_{10} \%$, which can be used as a measure of the autoconversion timescale. Then, we can define the turbulence enhancement factor, $E_{\mathrm{turb}}$, as

$E_{\text {turb }}=\frac{\left.P_{\text {auto }}\right|_{\mathrm{T}}}{\left.P_{\text {auto }}\right|_{\mathrm{NoT}}}=\frac{\overline{t_{10 \%}} \mathrm{NoT}}{\overline{t_{10 \%}}}$,

where the overbar indicates the mean value.

Figure 9a shows $E_{\text {turb }}$ as a function of $\epsilon$ for $R e_{\lambda}=66$ in the $r_{\mathrm{m} 0}=10 \mu \mathrm{m}$ case. The LCS data show an almost linear increase with increasing $\epsilon$. Both the SCE simulation with the Ayala-Wang kernel (SCE-Ayala hereafter) and that with the Onishi kernel (SCE-Onishi hereafter) show the same trend with the LCS data, although the SCE-Ayala slightly over- estimates the enhancement. The maximum relative difference between the SCE-Ayala and SCE-Onishi kernels was as small as $22 \%$ at $\epsilon=500 \mathrm{~cm}^{2} \mathrm{~s}^{-3}$. Both the SCE-Ayala and the SCE-Onishi kernels show a kink at $\epsilon=600 \mathrm{~cm}^{2} \mathrm{~s}^{-3}$, where the turbulence enhancement on collision efficiency levels off. Figure $9 \mathrm{~b}$ shows $E_{\text {turb }}$ as a function of $R e_{\lambda}$ for $\epsilon=$ $400 \mathrm{~cm}^{2} \mathrm{~s}^{-3}$ in the case of $r_{\mathrm{m} 0}=10 \mu \mathrm{m}$. The SCE-Ayala and the SCE-Onishi kernels show different trends: the SCE-Ayala predicts an increasing enhancement with increasing $R e_{\lambda}$, while the SCE-Onishi predicts almost constant or slightly decreasing enhancement. The difference between the two SCE predictions becomes larger for larger $R e_{\lambda}$, with the LCS result closer to the SCE-Onishi prediction. The difference between the SCE-Ayala and the SCE-Onishi kernels can be explained by the Reynolds-number dependence of the two kernels, as discussed in Sect. 4.4. This Reynolds-number dependence is relevant, because the SCE prediction becomes very different at large $R e_{\lambda}$. For example, at $R e_{\lambda}=2 \times 10^{4}$, the SCE-Ayala prediction is 2.5 times larger than the SCEOnishi prediction. The LCS results for $R e_{\lambda} \leq 206$ support the SCE-Onishi prediction.

Figure 10 shows $E_{\text {turb }}$ for the $r_{\mathrm{m} 0}=15 \mu \mathrm{m}$ case, which was also discussed in Onishi et al. (2015). This study additionally performed the simulation for $R e_{\lambda}=333$ to investigate the Reynolds-number dependence more clearly. Basically, the results are similar to those in the previous fig- 

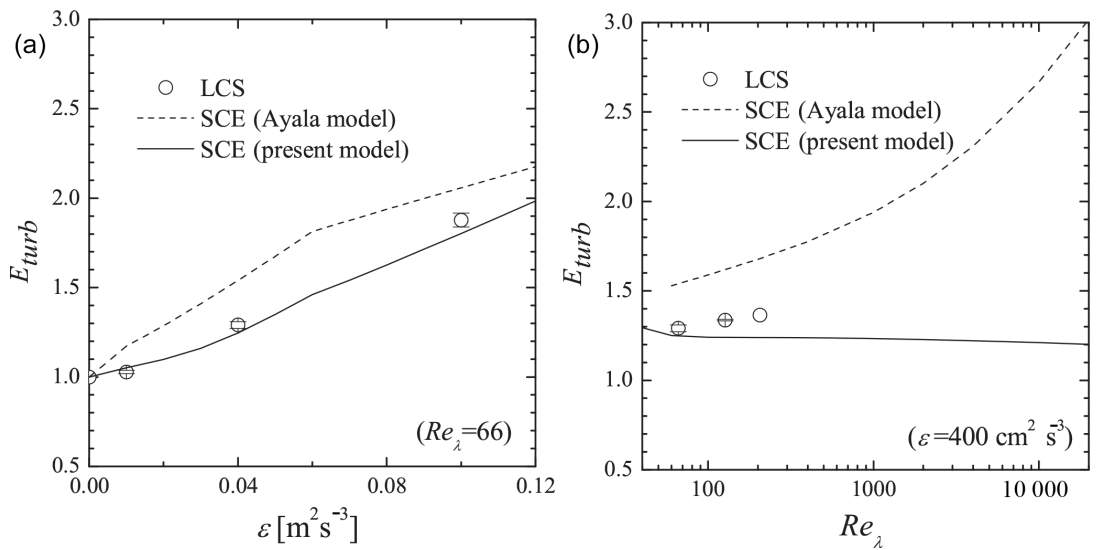

Figure 9. Turbulence enhancement factors for $\overline{r_{c}}=10 \mu \mathrm{m}$ as a function of (a) the energy dissipation rate $\epsilon$ and (b) the Taylor-microscalebased Reynolds number $R e_{\lambda} \cdot R e_{\lambda}=66$ in (a) and $\epsilon=400 \mathrm{~cm}^{2} \mathrm{~s}^{-3}$ in (b). The error bars indicate the standard deviations.

ure. In Fig. 10, the SCE-Ayala and the SCE-Onishi kernels show closer results for $R e_{\lambda}=66$, and both SCE-Ayala and SCE-Onishi slightly overestimate the enhancement for $\epsilon>400 \mathrm{~cm}^{2} \mathrm{~s}^{-3}$. The difference between the two predictions at $R e_{\lambda}=2 \times 10^{4}$ is larger: the SCE-Ayala prediction is 3.0 times larger than the SCE-Onishi prediction. The LCS results for $R e_{\lambda}$ up to 333 clearly support the SCE-Onishi prediction.

In summary, both Figs. 9 and 10 show that the SCE-Ayala and the SCE-Onishi kernels produce consistent results for low $R e_{\lambda}$ with about a $20 \%$ difference at most, but the two show very different values at large $R e_{\lambda}$ : the SCE-Ayala prediction becomes larger than the SCE-Onishi by a factor of up to 3 in cloud turbulence. This clearly suggests a strong demand for collision growth data with larger $R e_{\lambda}$ to construct a more robust turbulent kernel.

\subsection{Periodicity influence}

As noted in Woittiez et al. (2009) and discussed in Appendix A in Ireland et al. (2016b), the periodicity of the computational domain may lead to errors for the settling particles with large St. Ireland et al. (2016b) defined the critical St, $S t_{\text {crit }}$, as

$S t_{\text {crit }}=F r \frac{L}{l} \frac{u^{\prime}}{u_{\eta}}$,

where $\mathrm{Fr}$ is the Froude number $\left(=a_{\eta} / g\right.$, where $a_{\eta}$ is the Kolmogorov-scale acceleration), $L\left(=2 \pi L_{0}\right.$ in this study) is the domain size, $l$ is the integral scale, and $u_{\eta}$ is the Kolmogorov-scale velocity. For $S t$ larger than $S t_{\text {crit }}$, the periodicity problem may arise.

Figures 4,9 , and 10 are for settling particles. For those figures, we have calculated $S t_{\text {crit }}$ to check the periodicity problem. (i) For Fig. 4, $S t_{\text {crit }}=3.7$, which corresponds to $r_{\text {crit }}=75 \mu \mathrm{m} ; r_{\text {crit }}$ is the radius of particle with $S t=S t_{\text {crit }}$. The two plots from DNS, which correspond to $r_{2}=80 \mu \mathrm{m}$ and $120 \mu \mathrm{m}$, exceed $r_{\text {crit }}$. However, since the two plots are more or less similar with the gravitational (Hall) kernel values, the turbulent contribution would be small compared to the gravitational settling contribution. That is the error due to the periodicity would not significantly affect the results. (ii) For Figs. 9a and 10a, $r_{\text {crit }}$ are 50, 65, and $70 \mu \mathrm{m}$ for $\epsilon=100,400$, and $1000 \mathrm{~cm}^{2} \mathrm{~s}^{-3}$, respectively. For Figs. $9 \mathrm{~b}$ and $10 \mathrm{~b} r_{\text {crit }}$ are $65,75,85$, and $90 \mu \mathrm{m}$ for $R e_{\lambda}=66.1,127,206$, and 333, respectively. The enhancement factor $E_{\text {turb }}$, shown in Figs. 9 and 10 , was evaluated by $t_{10} \%$, which is defined as the time required for a cloud to convert $10 \%$ of its cloud mass into rain category drops. The threshold between cloud and rain categories was set at $r=40 \mu \mathrm{m}$. That is, $10 \%$ of particles, in mass and volume, are larger than $40 \mu \mathrm{m}$ in radius at $t=t_{10} \%$ by definition. For example, according to the DNS results, $3 \%$ of particles are larger than $50 \mu \mathrm{m}$ and only $0.9 \%$ of particles are larger than $60 \mu \mathrm{m}$ at $t=t_{10} \%$. The percentage of particles that are larger than $50 \mu \mathrm{m}$ in radius may have some impact on $t=t_{10} \%$ and consequently $E_{\text {turb. In this sense, the }}$ plot for $\epsilon=100 \mathrm{~cm}^{2} \mathrm{~s}^{-3}$ in Figs. 9a and 10a, whose $r_{\text {crit }}$ is $50 \mu \mathrm{m}$, may contain some error associated with the periodicity problem. However, since $E_{\text {turb }}$ for that plot is nearly unity, indicating small turbulence enhancement, the periodicity problem does not change the present findings.

\section{Conclusions}

This study investigated the Reynolds-number dependence of turbulence enhancement on the collision growth of cloud droplets. The Onishi turbulent coagulation kernel proposed in Onishi et al. (2015) was updated by using the present direct numerical simulation (DNS) results for the Taylormicroscale-based Reynolds number $\left(R e_{\lambda}\right)$ up to 1140 . The following three components were updated: (i) the radial distribution function at contact separation of a monodisperse suspension of droplets, i.e., the clustering effect, $g_{11}$; (ii) the 

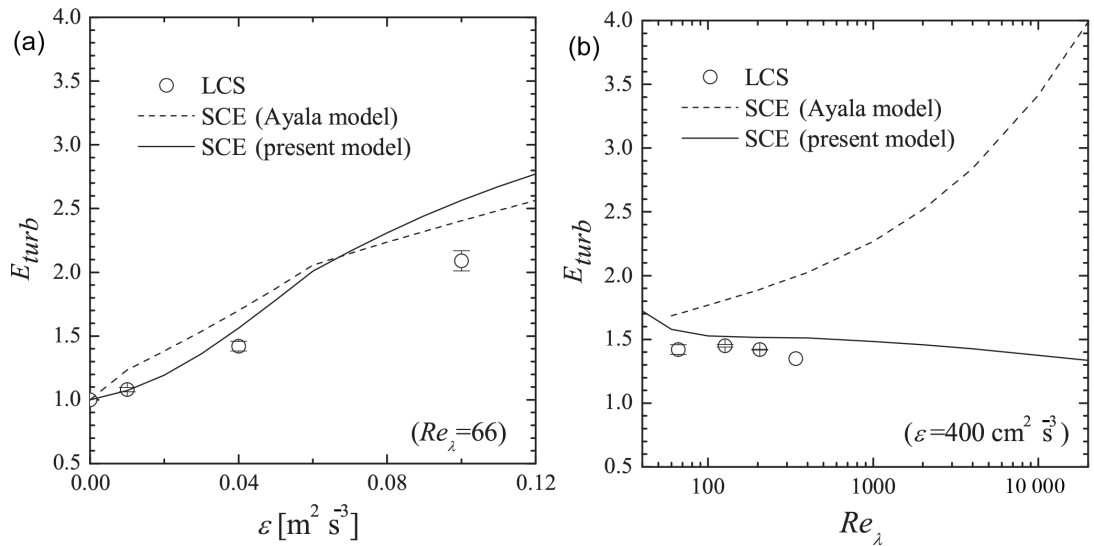

Figure 10. Turbulence enhancement factors for $\overline{r_{c}}=15$ as a function of (a) the energy dissipation rate and (b) the Taylor-microscale- based Reynolds number $R e_{\lambda} \cdot R e_{\lambda}=66$ in (a) and $\epsilon=400 \mathrm{~cm}^{2} \mathrm{~s}^{-3}$ in (b). The error bars indicate the standard deviations.

radial relative velocity at contact separation, $\left\langle\left|w_{\mathrm{r}}\right|\right\rangle$; and (iii) the turbulence enhancement on collision efficiency, $\eta_{E}$.

We confirmed that the updated $g_{11}$ parameterization agrees better with DNS results than the original parameterization for $R e_{\lambda} \sim 100$. We also confirmed that the updated parameterization has better agreement with the Reynolds-number dependence of $g_{11}$ for the estimated values of $S t=0.4$ and 0.6. The model of radial relative velocity was updated to include the effect of the gravitational sedimentation of droplets. The comparison with the DNS results confirmed that the updated model for $\left\langle\left|w_{\mathrm{r}}\right|\right\rangle$ is better than the original one. The Onishi coagulation kernel employed the turbulence enhancement on collision efficiency $\eta_{E}$, tabulated in Wang et al. (2008). The updated kernel is intended to adjust to more recent $\eta_{E}$ values, tabulated in Wang and Grabowski (2009). It should be noted that the collision efficiency $E_{\mathrm{c}}$ in Pinsky et al. (2001) ( $\left.E_{\mathrm{c}, \mathrm{PKS} 01}\right)$, which the Onishi kernel employs, is different from the $E_{\mathrm{c}}$ in Hall (1980) ( $\left.E_{\mathrm{c}, \text { Hall }}\right)$, particularly for $r_{2} / r_{1} \sim 0$ or $\sim 1$. We proposed a compensation such that $\eta_{E}$ (in Wang and Grabowski, 2009), which shows the turbulence enhancement against $E_{\mathrm{c}, \text { Hall }}$, is applicable to the kernel with $E_{\mathrm{c}, \mathrm{PKS} 01}$. The proposed compensation is simply to multiply $\eta_{E}$ in Wang and Grabowski (2009) by $E_{\mathrm{c}, \mathrm{PKS} 01} / E_{\mathrm{c}, \text { Hall }}$.

The present Onishi coagulation kernel was compared with the Ayala-Wang kernel (Ayala et al., 2008a; Wang et al., 2008) together with the DNS values for $R e_{\lambda}=66$ and the energy dissipation rate $\epsilon=400 \mathrm{~cm}^{2} \mathrm{~s}^{-3}$. For $K_{\mathrm{coag}}\left(r_{1}=30 \mu \mathrm{m}\right.$, $r_{2}$ ), both kernels show similar values comparable to the DNS values except for $r_{2} \sim r_{1}$. For the nearly monodisperse case, the Ayala-Wang kernel overestimates the kernel but provides a sharp convex shape, i.e., a clear local maximum at $r_{2}=$ $30 \mu \mathrm{m}$, that agrees with the DNS data qualitatively. The Onishi kernel does not show such a convex shape due to weaker acceleration-driven diffusion on the clustering effect $g_{12}$, but the kernel values are in fairly good agreement with the DNS. The Reynolds-number dependence of the two kernels was also compared. It was shown that the Ayala-Wang kernel in- creases for the autoconversion region $\left(r_{1}, r_{2}<40 \mu \mathrm{m}\right)$ and the accretion region $\left(r_{1}<40\right.$ and $r_{2}>40$, and $r_{1}>40$ and $\left.r_{2}<40 \mu \mathrm{m}\right)$. In contrast, the Onishi kernel decreases for the autoconversion region but increases for the rain-rain selfcollection region $\left(r_{1}, r_{2}>40 \mu \mathrm{m}\right)$. These Reynolds-number dependences can be attributed to the Reynolds-number dependence of the clustering effect.

We also compared the stochastic collision-coalescence equation (SCE) simulations for both kernels; one with the Ayala-Wang kernel (SCE-Ayala) and the other with the present Onishi kernel (SCE-Onishi). Lagrangian Cloud Simulator (LCS; Onishi et al., 2015) simulations were also conducted to obtain reference data of the turbulent enhancement on collisional growth, in particular the enhancement on the autoconversion rate. The SCE-Ayala and SCE-Onishi kernels show consistent results for $R e_{\lambda}=66$ with about a $20 \%$ difference at most, but the two SCE simulations show a different Reynolds-number dependence, resulting in large differences at large $R e_{\lambda}$. It should be emphasized that the SCE-Ayala prediction can become larger than the SCE-Onishi by a factor of up to 3 in the typical large $R e_{\lambda}$ range observed in cloud turbulence. These simulations clearly suggest a strong demand for reference collision growth data with larger $R e_{\lambda}$ from DNS or laboratory measurement to construct a more robust kernel model. This is our goal in future studies.

\section{Data availability}

Data for the present graphs are available from the corresponding author upon request.

The Supplement related to this article is available online at doi:10.5194/acp-16-12441-2016-supplement. 
Acknowledgements. Part of the presented simulations were performed on the supercomputer Earth Simulator at the Japan Agency for Marine-Earth Science and Technology. The large-size simulations for collision statistics for $\operatorname{Re}_{\lambda}=874$ and 1140 were performed on the $\mathrm{K}$ computer provided by the RIKEN Advanced Institute for Computational Science through the HPCI System Research project (project ID: hp140120). We thank J. C. Vassilicos for his insightful comments on Sect. 4.1. We also thank Peter Ireland for providing the data for Fig. 2. We finally thank L.-P. Wang and one anonymous reviewer for valuable comments and discussion that helped to improve the paper.

Edited by: P. Chuang

Reviewed by: L.-P. Wang and one anonymous referee

\section{References}

Abrahamson, J.: Collision rates of small particles in a vigorously turbulent fluid, Chem. Eng. Sci., 30, 1371-1379, 1975.

Ayala, O., Grabowski, W. W., and Wang, L.-P.: A hybrid approach for simulating turbulent collisions of hydrodynamicallyinteracting particles, J. Comput. Phys., 225, 51-73, 2007.

Ayala, O., Rosa, B., and Wang, L.-P.: Effects of turbulence on the geometric collision rate of sedimenting droplets. Part 2. Theory and parameterization, New J. Phys., 10, 075016, doi:10.1088/1367-2630/10/7/075016, 2008a.

Ayala, O., Rosa, B., Wang, L.-P., and Grabowski, W. W.: Effects of turbulence on the geometric collision rate of sedimenting droplets. Part 1. Results from direct numerical simulation, New J. Phys., 10, 075015, doi:10.1088/1367-2630/10/7/075015, 2008b.

Blyth, A. M.: Entrainment in cumulus clouds, J. Appl. Meteorol., 32, 626-641, 1993.

Chun, J., Koch, D. L., Rani, S. L., Ahluwalia, A., and Collins, L. R.: Clustering of aerosol particles in isotropic turbulence, J. Fluid Mech., 536, 219-251, doi:10.1017/S0022112005004568, 2005.

Coleman, S. W. and Vassilicos, J. C.: A unified sweep-stick mechanism to explain particle clustering in two- and three-dimensional homogeneous, isotropic turbulence, Phys. Fluids, 21, 113301, doi:10.1063/1.3257638, 2009.

Dallas, V. and Vassilicos, J. C.: Rapid growth of cloud droplets by turbulence, Physical Rev. E, 84, 1-5, doi:10.1103/PhysRevE.84.046315, 2011.

Derevyanko, S., Falkovich, G., and Turitsyn, S.: Evolution of nonuniformly seeded warm clouds in idealized turbulent conditions, New J. Phys., 10, 075019, doi:10.1088/1367-2630/10/7/075019, 2008.

Dodin, Z. and Elperin, T.: On the collision rate of particles in turbulent flow with gravity, Phys. Fluids, 14, 2921, doi:10.1063/1.1490136, 2002.

Falkovich, G. and Pumir, A.: Sling Effect in Collisions of Water Droplets in Turbulent Clouds, J. Atmos. Sci., 64, 4497-4505, doi:10.1175/2007JAS2371.1, 2007.

Grabowski, W. W. and Wang, L.-P.: Growth of Cloud Droplets in a Turbulent Environment, Annu. Rev. Fluid Mech., 45, 293-324, 2013.

Hall, W. D.: A Detailed Microphysical Model Within a TwoDimensional Dynamic Framework: Model Description and Preliminary Results, J. Atmos. Sci., 37, 2486-2507, 1980.
Hirt, C. W. and Cook, J. L.: Calculating three-dimensional flows around structures and over rough terrain, J. Comput. Phys., 10, 324-340, 1972.

Ireland, P. J., Bragg, A. D., and Collins, L. R.: The effect of Reynolds number on inertial particle dynamics in isotropic turbulence. Part 1. Simulations without gravitational effects, J. Fluid Mech., 796, 617-658, doi:10.1017/jfm.2016.238, 2016a.

Ireland, P. J., Bragg, A. D., and Collins, L. R.: The effect of Reynolds number on inertial particle dynamics in isotropic turbulence. Part 2. Simulations with gravitational effects, J. Fluid Mech., 796, 659-711, doi:10.1017/jfm.2016.227, 2016b.

Kolmogorov, A.: A refinement of previous hypotheses concerning the local structure of turbulence in a visous incompressible fluid at high Reynolds number, J. Fluid Mech., 13, 82-85, 1962.

Krueger, S. K., Su, C. W., and McMurtry, P. A.: Modeling entrainment and finescale mixing in cumulus clouds, J. Atmos. Sci., 54, 2697-2712, 1997.

Kruis, F. and Kusters, K.: THE COLLISION RATE OF PARTICLES IN TURBULENT FLOW, Chem. Eng. Commun., 158, 201-230, doi:10.1080/00986449708936589, 1997.

Maxey, M. R.: The gravitational settling of aerosol particles in homogeneous turbulence and random flow fields, J. Fluid Mech., 174, 441-465, 1987.

Morinishi, Y., Lund, T. S., Vasilyev, O. V., and Moin, P.: Fully conservative higher order finite difference schemes for incompressible flow, J. Comput. Phys., 143, 90-124, 1998.

Onishi, R. and Takahashi, K.: A Warm-Bin-Cold-Bulk Cloud Microphysical Model, J. Atmos. Sci., 69, 1474-1497, 2012.

Onishi, R. and Vassilicos, J. C.: Collision statistics of inertial particles in two-dimensional homogeneous isotropic turbulence with an inverse cascade, J. Fluid Mech., 745, 279-299, 2014.

Onishi, R., Takahashi, K., and Komori, S.: Influence of gravity on collisions of monodispersed droplets in homogeneous isotropic turbulence, Phys. Fluids, 21, 125108, doi:10.1063/1.3276906, 2009.

Onishi, R., Baba, Y., and Takahashi, K.: Large-scale forcing with less communication in finite-difference simulations of stationary isotropic turbulence, J. Comput. Phys., 230, 4088-4099, doi:10.1016/j.jcp.2011.02.034, 2011.

Onishi, R., Takahashi, K., and Vassilicos, J. C.: An efficient parallel simulation of interacting inertial particles in homogeneous isotropic turbulence, J. Comput. Phys., 242, 809-827, 2013.

Onishi, R., Matsuda, K., and Takahashi, K.: Lagrangian Tracking Simulation of Droplet Growth in Turbulence - Turbulence Enhancement of Autoconversion Rate, J. Atmos. Sci., 72, 25912607, 2015.

Pinsky, M., Khain, A., and Shapiro, M.: Collision Efficiency of Drops in a Wide Range of Reynolds Numbers: Effects of Pressure on Spectrum Evolution, Journal of the Atmospheric Sciences, 58, 742-764, 2001.

Pope, S.: Turbulent Flows, Cambridge University Press, Cambridge, UK, 2000.

Rowe, P. N. and Henwood, G. A.: Drag forces in a hydraulic model of a fluidised bed - Part I., T. I. Chem. Eng.-Lond., 39, 43-54, 1961.

Saffman, P. G. and Turner, J. S.: On the collision of drops in turbulent clouds, J. Fluid Mech., 1, 16-30, doi:10.1017/S0022112056000020, 1956. 
Saw, E. W., Shaw, R. A., Ayyalasomayajula, S., Chuang, P. Y., and Gylfason, A.: Inertial Clustering of Particles in HighReynolds-Number Turbulence, Phys. Rev. Lett., 100, 214501, doi:10.1103/PhysRevLett.100.214501, 2008.

Seifert, A., Nuijens, L., and Stevens, B.: Turbulence effects on warm-rain autoconversion in precipitating shallow convection, Q. J. Roy. Meteor. Soc., 136, 1753-1762, doi:10.1002/qj.684, 2010.

Sidin, R. S. R., Ijzermans, R. H. A., and Reeks, M. W.: A Lagrangian approach to droplet condensation in atmospheric clouds, Phys. Fluids, 21, 106603, doi:10.1063/1.3244646, 2009.

Soong, S. T.: Numerical Simulation of Warm Rain Development in an Axisymmetric Cloud Model, J. Atmos. Sci., 31, 1262-1285, 1974.

Sundaram, S. and Collins, L.: Numerical considerations in simulating a turbulent suspension of finite-volume particles, J. Comput. Phys., 124, 337-350, 1996.

Sundaram, S. and Collins, L. R.: Collision statistics in an isotropic particle-laden turbulent suspension. Part 1. Direct numerical simulations, J. Fluid Mech., 335, 75-109, 1997.

Van Den Heever, S. C. and Cotton, W. R.: Urban Aerosol Impacts on Downwind Convective Storms, J. Appl. Meteorol. Clim., 46, 828-850, doi:10.1175/JAM2492.1, 2007.

Wang, L.-P. and Grabowski, W. W.: The role of air turbulence in warm rain initiation, Atmos. Sci. Lett., 10, 1-8, doi:10.1002/asl.210, 2009.
Wang, L.-P., Wexler, A. S., and Zhou, Y.: Statistical mechanical descriptions of turbulent coagulation, Phys. Fluids, 10, 2647-2651, doi:10.1063/1.869777, 1998.

Wang, L.-P., Wexler, A. S., and Zhou, Y.: Statistical mechanical description and modelling of turbulent collision of inertial particles, J. Fluid Mech., 415, 117-153, 2000.

Wang, L.-P., Ayala, O., Rosa, B., and Grabowski, W. W.: Turbulent collision efficiency of heavy particles relevant to cloud droplets, New J. Phys., 10, 075013, doi:10.1088/1367-2630/10/7/075013, 2008.

Woittiez, E. J. P., Jonker, H. J. J., and Portela, L. M.: On the Combined Effects of Turbulence and Gravity on Droplet Collisions in Clouds: A Numerical Study, J. Atmos. Sci., 66, 1926-1943, doi:10.1175/2005JAS2669.1, 2009.

Yin, Y., Levin, Z., Reisin, T. G., and Tzivion, S.: The effects of giant cloud condensation nuclei on the development of precipitation in convective clouds - a numerical study, Atmos. Res., 53, 91-116, doi:10.1016/S0169-8095(99)00046-0, 2000.

Zaichik, L. and Alipchenkov, V.: Statistical models for predicting pair dispersion and particle clustering in isotropic turbulence and their applications, New J. Phys., 11, 3018, doi:10.1088/13672630/11/10/103018, 2009.

Zhou, Y., Wexler, A. S., and Wang, L.-P.: Modelling turbulent collision of bidisperse inertial particles, J. Fluid Mech., 433, 77-104, 2001. 